\title{
Sentinel-4: a geostationary imaging UVN spectrometer for air quality monitoring: integration and alignment of the instrument proto flight model
}

Pierangelo Marenaci, Luis Estares Otal, Ellart Meijer, Jean-Francois Pittet, Stefan Riedl, et al.

Pierangelo Marenaci, Luis Estares Otal, Ellart Meijer, Jean-Francois Pittet, Stefan Riedl, Sai Shilpa Suram, Christoph Ehrhart, Grégory Bazalgette Courrèges-Lacoste, Gerard Bulsa, Arnaud Pasquet, Giorgio Bagnasco, "Sentinel-4: a geostationary imaging UVN spectrometer for air quality monitoring: integration and alignment of the instrument proto flight model," Proc. SPIE 11852, International Conference on Space Optics - ICSO 2020, 1185267 (11 June 2021); doi: 10.1117/12.2600228

SPIE. Event: International Conference on Space Optics - ICSO 2021, 2021, Online 


\section{International Conference on Space Optics-ICSO 2020}

Virtual Conference

30 March-2 April 2021

Edited by Bruno Cugny, Zoran Sodnik, and Nikos Karafolas
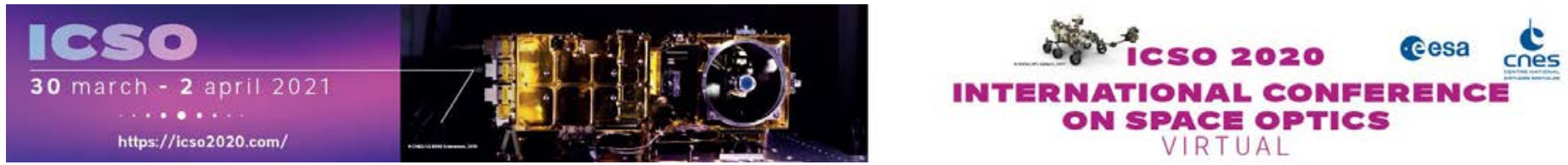

Sentinel-4 - A Geostationary Imaging UVN Spectrometer for Air Quality Monitoring - Integration and Alignment of the Instrument Proto Flight Model

\section{Cesa issopocestings denes}




\title{
Sentinel-4 - A Geostationary Imaging UVN Spectrometer for Air Quality Monitoring - Integration and Alignment of the Instrument Proto Flight Model
}

\author{
Pierangelo Marenaci ${ }^{\mathrm{a}}$ (pierangelo.marenaci@airbus.com), Luis Esteras Otal ${ }^{\mathrm{a}}$ \\ (luis.esteras.otal@airbus.com), Ellart Meijer ${ }^{\mathrm{a}}$ (ellart.meijer@airbus.com), Jean-Francois Pittet ${ }^{\mathrm{a}}$ (jean- \\ francois.pittet@airbus.com), Stefan Riedl ${ }^{\mathrm{a}}$ (stefan.riedl@airbus.com), Sai Shilpa Suram ${ }^{\mathrm{a}}$ \\ (sai_shilpa.suram.external@airbus.com),Christoph Ehrhart (christoph.ehrhart@airbus.com), \\ Grégory Bazalgette Courrèges-Lacoste ${ }^{\mathrm{b}}$ (gregory.bazalgette@esa.int), Gerard Bulsa ${ }^{\mathrm{b}}$ \\ (Gerard.Bulsa@esa.int), Arnaud Pasquet ${ }^{\mathrm{b}}$ (arnaud.pasquet@esa.int), Giorgio Bagnasco ${ }^{\mathrm{b}}$ \\ (giorgio.bagnasco@esa.int) \\ ${ }^{a}$ Airbus Defence and Space GmbH, Taufkirchen, Germany \\ ${ }^{b}$ European Space Agency ESTEC, Noordwijk, Netherlands
}

\begin{abstract}
Sentinel-4 is an imaging UVN (UV-VIS-NIR) spectrometer, developed by Airbus Defence and Space GmbH under ESA contract in the frame of the joint EU/ESA COPERNICUS program. The mission objective is the operational monitoring of trace gas concentrations for atmospheric chemistry and climate over Europe. Sentinel-4 will provide accurate measurements of key atmospheric constituents such as ozone, nitrogen dioxide, sulfur dioxide, methane, and aerosol
\end{abstract} properties.

The instrument is a hyperspectral imager. It is composed of two imaging spectrometers covering the spectral ranges of $305-500 \mathrm{~nm}$ and $750-775 \mathrm{~nm}$ and a telescope which projects an image of the Earth onto the slits of the spectrometers. The instrument will be placed in a geostationary orbit, and a 3D spectral-spatial data-cube will be acquired thanks to the mirror scanning in East-West direction.

The Telescope Spectrograph Assembly (TSA) represents the core of the optical system inside the Optical Instrument Module (OIM). It is composed of one common Telescope, two Spectrographs and two Focal Plane Assemblies integrated and aligned into a three-dimensional supporting structure. The TSA is later integrated into the main instrument structure which already includes the Scan Mirror Unit, Calibration Assembly and Front Baffle; thus completing the full optical chain.

This paper gives an overview of the TSA integration and alignment activities for the Proto Flight Model (PFM) performed at the Airbus premises in Ottobrunn, Germany. It describes the alignment philosophy developed to meet the challenging optical requirements, including for example, the spatial co-registration between the two spectrometers and the spatial sampling distance on ground, after the transition from ambient laboratory conditions to the instrument operating conditions in geostationary orbit.

The results of the optical tests in operating conditions produced by the Optical Ground Support Equipment are also included and discussed in this paper.

\section{INTRODUCTION}

Sentinel-4 is an imaging UVN (UV-VIS-NIR) spectrometer which will provide accurate measurements of key atmospheric constituents such as ozone, nitrogen dioxide, sulfur dioxide, methane, and aerosol properties over Europe and adjacent regions from a geostationary orbit (see Figure 1) - hence the motto of Sentinel-4 "Knowing what we breath". 
In the family of already flown UVN spectrometers (SCIAMACHY, OMI, GOME \& GOME 2) and of those spectrometers recently launched (TROPOMI) and currently under development (Sentinel-5), Sentinel 4 is unique in being the first geostationary UVN mission, together with very similar geostationary UVN missions over other continents, which are being developed in parallel by NASA (TEMPO) and KARI (GEMS). Furthermore, thanks to its 60-minutes repeat cycle measurements and high spatial resolution $\left(8 \times 8 \mathrm{~km}^{2}\right)$. Sentinel-4 will increase the frequency of cloud-free observations, which is necessary to assess troposphere variability.

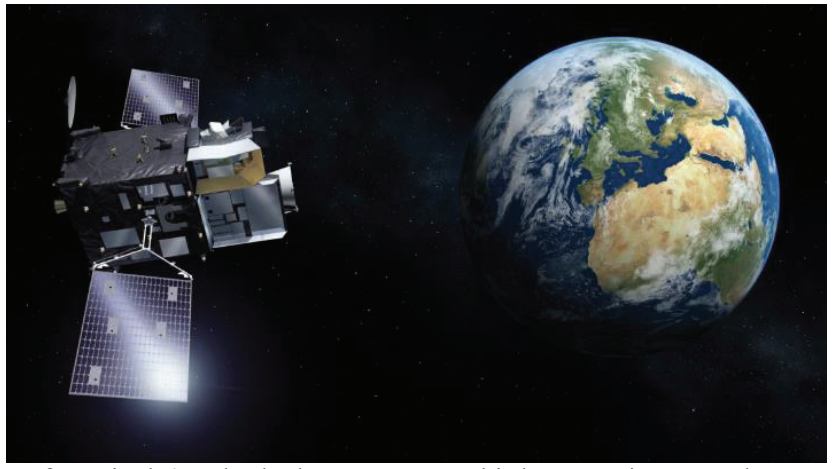

Figure 1. Artistic impression of Sentinel-4 embarked on Meteosat Third Generation-Sounder.

Two identical Sentinel-4 instruments (PFM and FM-2) will be embarked, as Customer Furnished Item (CFI), fully verified, qualified and calibrated respectively onto two EUMETSAT satellites: Meteosat Third Generation-Sounder 1 \& 2 (MTG-S1 and MTG-S2).

The spatial coverage over Europe and adjacent regions will be achieved by continuous East/West scanning of the image by a push-broom mirror mechanism, which will cover a field-of-regard of about 11 degrees, while the North/South instantaneous field-of-view (IFOV) will be equal to about 4 degrees.

Blue and red lines, shown in Figure 2, indicate the borders of the specified Geo-Coverage area (GCA), which is the total area to be covered every day. The overall daily Earth observation pattern consists of a series of 1 hour-long East-to-West scans ("repeat cycles") with a fast West-to-East retrace in-between. The green border indicates the size of a 1-hour repeat cycle (Reference Coverage - RA). Depending on the seasonally varying duration of Earth illumination by the Sun, the daily Earth observation scan series consists of 16 (winter) to 20 (summer) 1h-scans.

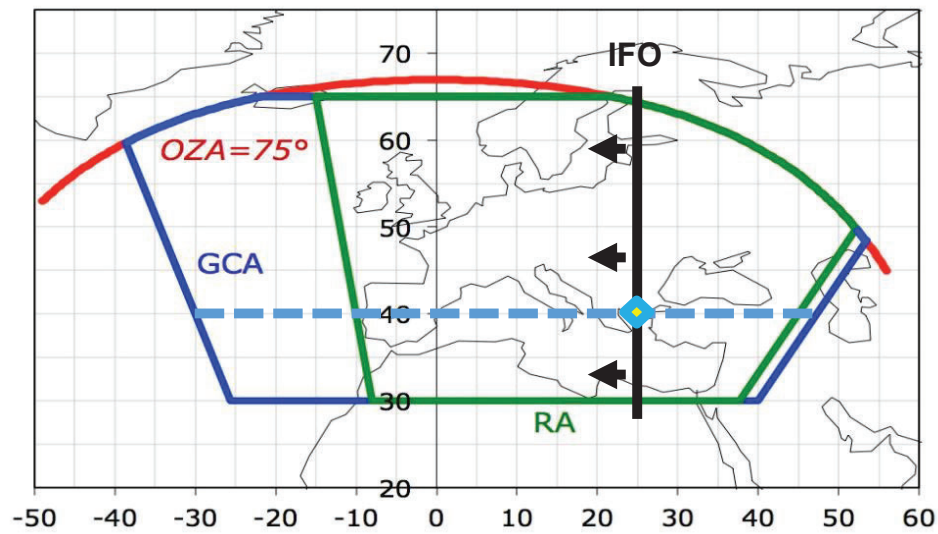

Figure 2. Earth coverage from the geostationary Sentinel-4 perspective.

While observing Europe and its adjacent regions, the Sentinel-4 imaging spectrometer will acquire continuous spectra of Earth radiance using the Sun as a light source illuminating the Earth. It will cover the Ultra Violet (305-400 nm), the 
Visible $(400-500 \mathrm{~nm})$ and the Near Infrared $(750-775 \mathrm{~nm})$ wavelength bands, with spectral resolution of $0.5 \mathrm{~nm}$ in the first two bands and $0.12 \mathrm{~nm}$ in the third band.

The instrument measurement concept, illustrated in Figure 3, can be described as follows:

- A scanning mirror (not shown in the figure), operating in push-broom mode, selects a strip of land whose "white light", reflected by the Earth and transmitted through the Earth atmosphere, is collected by the telescope.

- The collected "white light" is split into two 2 wavelength ranges (i.e. the Ultraviolet \& Visible-UVVIS and the Near-Infrared-NIR ranges) by a dichroic beam-splitter mirror and focused onto the two slits of the two separate spectrometers.

- Afterwards the light is first collimated onto the dispersing optical elements of the two spectrometer channels (either a grating or a grism) and dispersed in the spectral direction.

- The generated spectra are re-imaged onto two 2-dimensional charge coupled device (CCD) detector arrays. One dimension features the spectrum, the other dimension the spatial (North/South) direction corresponding to the selected strip on Earth (ground swath).

- At the end of this scanning process which lasts about 1 hour, spectra of multiple strips of land, which make up the complete field of view, are acquired and a complete spectral image of the Earth atmosphere over Europe is created. The process is repeated daily n-times so long as the relevant strip of land is illuminated by the Sun.

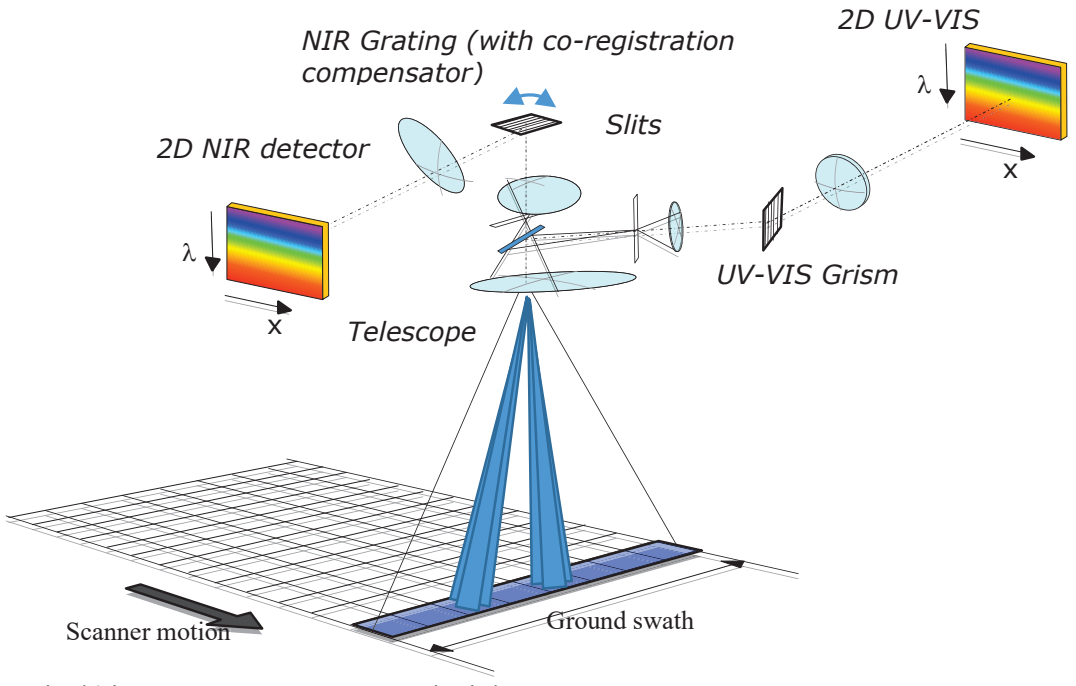

Figure 3. Sentinel4 instrument measurement principle.

The Sentinel-4 core optical design is shown in Figure 4. It consists of the following optical modules, designed to be independently manufactured and aligned: Scanner, Telescope Assembly including beamsplitter \& slits (TA), UVVIS and NIR Spectrograph Assemblies (USA and NSA), UV-VIS and NIR Focal Plane Assemblies (FPA). The main end-to-end performances driving the optical design are the polarization (polarization sensitivity and its spectral \& spatial features), the straylight and the co-registration.

Since the system level spatial co-registration requirements are defined on an absolute and not on a knowledge accuracy basis, very good co-registration has to be achieved by design. For the optical design this means ultra-low geometrical distortion and also extremely good matching of the effective focal lengths of the UV-VIS and the NIR optical path. 

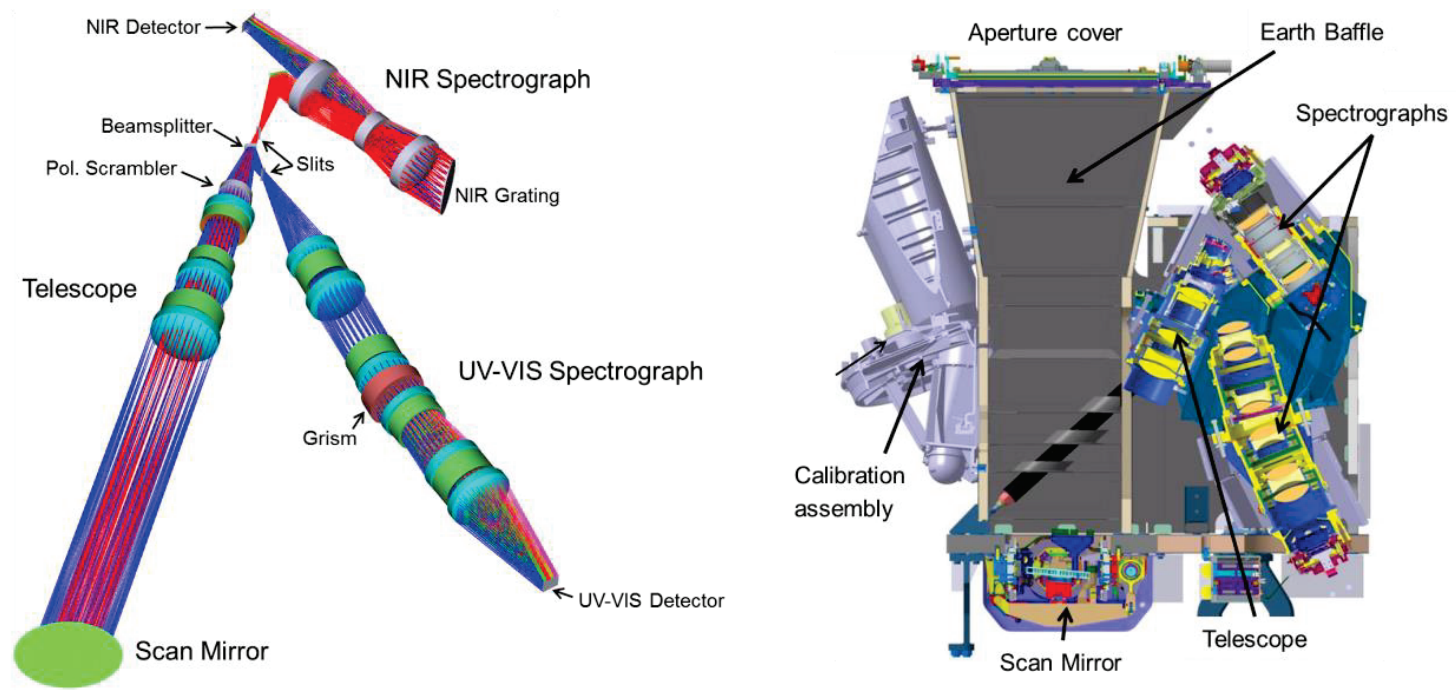

Figure 4. Left: TSA optical layout. Right: Opto-mechanical layout of the Optical Instrument Module (OIM).

The planar symmetry of the core optics, the on-axis lenses, a general optimization for low angles of incidence (e.g. on scanner) and low angles of dispersion, allow achieving almost neutral polarization behavior by design. These optical architecture features are also enabling factors for the low optical distortion. Since some optical elements still inevitably are polarization sensitive and show spectral features (e.g. grating and grism) a depolarizing element, the polarization scrambler, is introduced before these elements in the optical path. The pre-optimization of the optical architecture towards low polarization effects has two advantages regarding this polarization scrambler: 1) the front optics, including scan mirror and telescope optics, features sufficiently low polarization effects that the scrambler can be introduced after these elements. This leads to a significantly smaller scrambler, which has great advantages in terms of manufacturability. 2) A rather weakly depolarizing scrambler, thus associated with only small degradation of the optical point-spreadfunction (i.e. image quality), can still meet the system level polarization requirements.

Table 1: Main design and performance parameters of SENTINEL 4

\begin{tabular}{|c|c|c|c|}
\hline \multicolumn{4}{|c|}{ Spectral } \\
\hline Parameter & UV-VIS values & NIR values & Comments \\
\hline Wavelength range & $305-500 \mathrm{~nm}$ & $750-775 \mathrm{~nm}$ & \\
\hline $\begin{array}{l}\text { Spectral Resolution / } \\
\text { Spectral Oversampling }\end{array}$ & $\begin{array}{l}0.5 \mathrm{~nm} / \\
3\end{array}$ & $\begin{array}{c}0.12 \mathrm{~nm} / \\
3\end{array}$ & $\begin{array}{l}\text { Oversampling is Resolution divided by } \\
\text { spectral pixel sampling }\end{array}$ \\
\hline Spectral Calibration Accuracy & $0.0017 \mathrm{~nm}$ & $0.0020 \mathrm{~nm}$ & \\
\hline \multicolumn{4}{|c|}{ Geometric and Temporal Coverage } \\
\hline Parameter & \multicolumn{2}{|c|}{ Value(s) } & Comments \\
\hline Spatial Sampling Distance (SSD) & \multicolumn{2}{|c|}{ 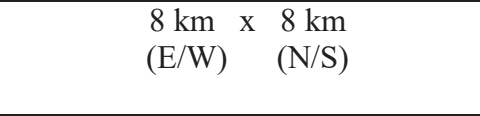 } & $\begin{array}{l}\text { On-ground-projected SSD at reference } \\
\text { point in Europe }\left(45^{\circ} \mathrm{N} \text { latitude; sub- }\right. \\
\text { satellite-point longitude) }\end{array}$ \\
\hline Integrated Energy & \multicolumn{2}{|c|}{$\begin{array}{l}70 \% \text { over } 1.47 \mathrm{SSD}_{\mathrm{EW}} * 1.13 \mathrm{SSD}_{\mathrm{NS}} \\
90 \% \text { over } 1.72 \mathrm{SSD}_{\mathrm{EW}} * 1.72 \mathrm{SSD}_{\mathrm{NS}}\end{array}$} & $\begin{array}{l}\text { Integrated energy is a measure for the } \\
\text { spatial resolution of the instrument }\end{array}$ \\
\hline N/S slit FOV (swath) & \multicolumn{2}{|c|}{$4.0^{\circ}$} & \\
\hline E/W coverage \& Repeat cycle & \multicolumn{2}{|c|}{ See Figure 2} & See Figure 2 \\
\hline Daily Earth observation time & $\begin{array}{l}\text { Summer max: } \\
\text { Winter min: }\end{array}$ & $\begin{array}{l}01: 40-21: 40 \\
03: 40-19: 40\end{array}$ & $\begin{array}{l}\text { Adjusted to seasonally varying solar } \\
\text { Earth illumination on monthly basis }\end{array}$ \\
\hline Spatial co-registration & $\begin{array}{l}\text { Intra-detecto } \\
\text { Inter-detecto }\end{array}$ & $\begin{array}{l}10 \% \text { of SSD } \\
20 \% \text { of SSD }\end{array}$ & $\begin{array}{c}\text { 2-dimensional }(\mathrm{E} / \mathrm{W} \& \mathrm{~N} / \mathrm{S}) \text { absolute co- } \\
\text { registration }\end{array}$ \\
\hline
\end{tabular}




\section{ALIGNMENT STRATEGY}

The Telescope Spectrograph Assembly (TSA) consists of three Combined Optical Assemblies (COA) and two Focal Plane Assemblies (FPA) mounted on a three dimensional aluminum structure (Figure 5). In total there are five interfaces that need to be aligned in six degrees of freedom by means of alignment shims. At each interface three degrees of freedom (thickness and tip/tilt angle) are defined by the thickness and wedge of the relative shim that is manufactured with a typical accuracy of $5 \mu \mathrm{m}$. The remaining three degrees of freedom (lateral displacement and clocking angle) are adjusted with the same level of accuracy within the range defined by the oversize of the bolts holes. Such accurate positioning is made possible by the use of pretension and alignment tools, specifically designed for each interface. The relative position of each optical assembly is measured by means of theodolites and laser trackers with an accuracy close to $5 \mu \mathrm{m}$ for displacements and 1 " for angles.

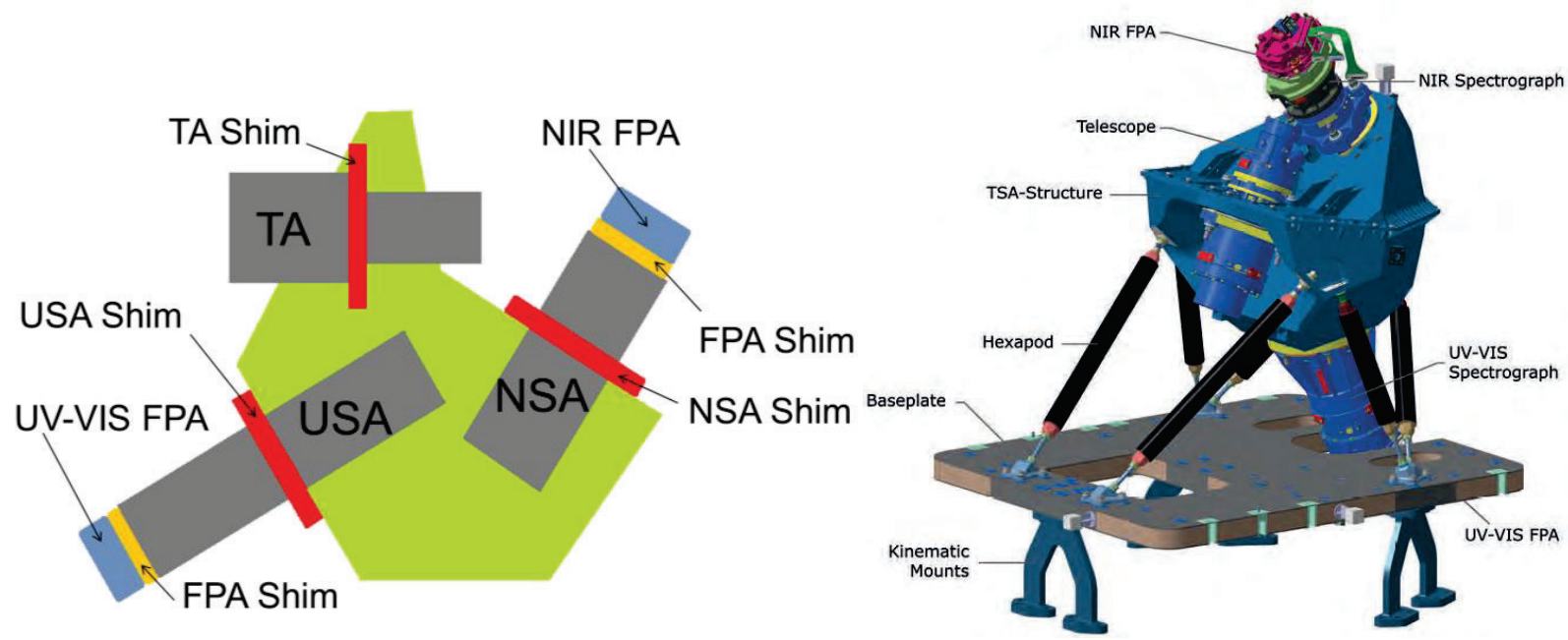

Figure 5. On the left side schematic view of the TSA with the five assemblies to be aligned and the relative alignment shims. The three COAs are grey, the two FPAs are blue. The red shims are used in the first part of the alignment, yellow shims in the second part. On the right side a mechanical drawing of the TSA mounted on the instrument baseplate.

One of the challenges of the TSA alignment is that the dioptric lens design of telescope and spectrometers in laboratory conditions behaves differently than in operating conditions in vacuum. The reason is the variable refractive index of air, dependent on environmental parameters like pressure, temperature and humidity. The main effect can be described by a geometrical shift in the position of object and image planes, but also a change of other optical parameters such as focal length and magnification. All optical measurements in air require therefore a compensation of the air-to-vacuum shift and the alignment status needs to be verified in vacuum. A specific Optical Ground Support Equipment (OGSE) was developed for the TSA alignment and provides all the necessary capabilities.

\subsection{Optical assemblies description}

The three optical assemblies and the two FPAs being aligned in the TSA are built by the subcontractor Jena Optronik as independent units. The Telescope Assembly (TA) comprises the main telescope objective, a polarization scrambler, a dichroic beam splitter and two separate slits (Figure 6). 

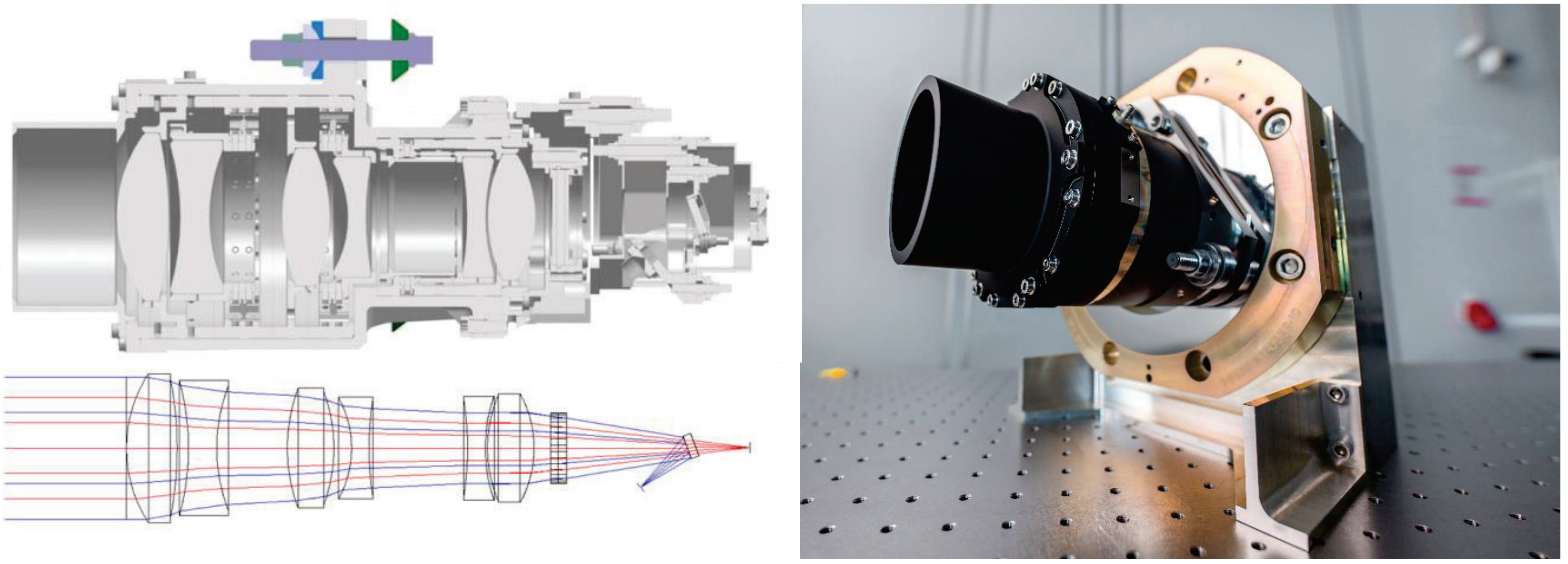

Figure 6. Telescope Assembly (TA). On the left side the mechanical cross section (top view) and the optical layout (bottom view). The blue rays define the UV-VIS path which is reflected by the dichroic beamsplitter, the red rays define the transmitted NIR path. The polarization scrambler is the last element before the beamsplitter. On the right side a picture of the optical assembly (image credit: Florian Brill for Jena Optronik GmbH).

The UV-VIS Spectrograph Assembly (USA) comprises a collimator objective, a grism as dispersive element and a camera objective (Figure 7). Like the TA, also the USA optical design consists of elements made of $\mathrm{CaF} 2$ and $\mathrm{SiO}$, mounted on passively actuated compensators that reduce the thermal sensitivity. This feature ensures not only a stable performance in orbit but also a lower sensitivity to thermal fluctuations during integration.

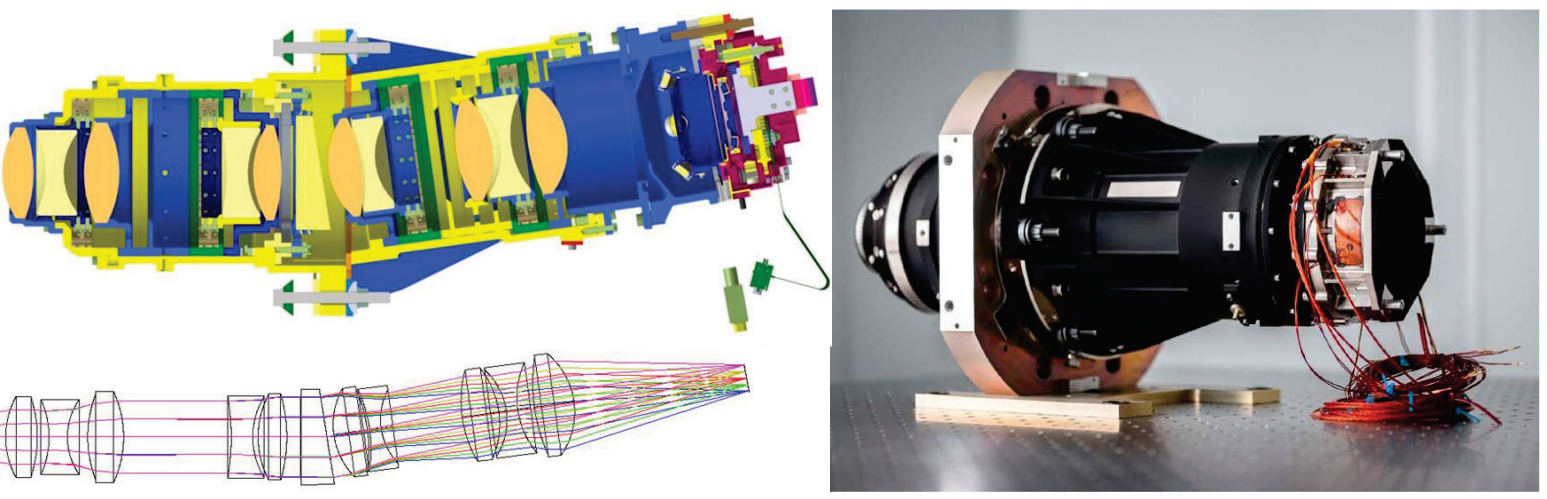

Figure 7. UV-VIS Spectrograph Assembly (USA) with UV-VIS FPA. On the left side the mechanical cross section (top view) and the optical layout (bottom view). The ray colors correspond to different wavelengths. On the right side a picture of the optical assembly without FPA (image credit: Florian Brill for Jena Optronik GmbH).

The NIR Spectrograph Assembly (NSA) comprises a single triplet objective used in double pass as collimator and camera plus a reflective grating as dispersive element operated close to the Littrow configuration (Figure 8). The reflective grating is mounted inside a mechanical assembly that has the capability to actuate a controlled tilt of the element thus shifting the image projected onto the FPA in spatial direction. This active compensator is used to align the pointing direction between the UV-VIS and NIR channels at subpixel level, thus simplifying the alignment and the compensation of ground-to-orbit effects. 

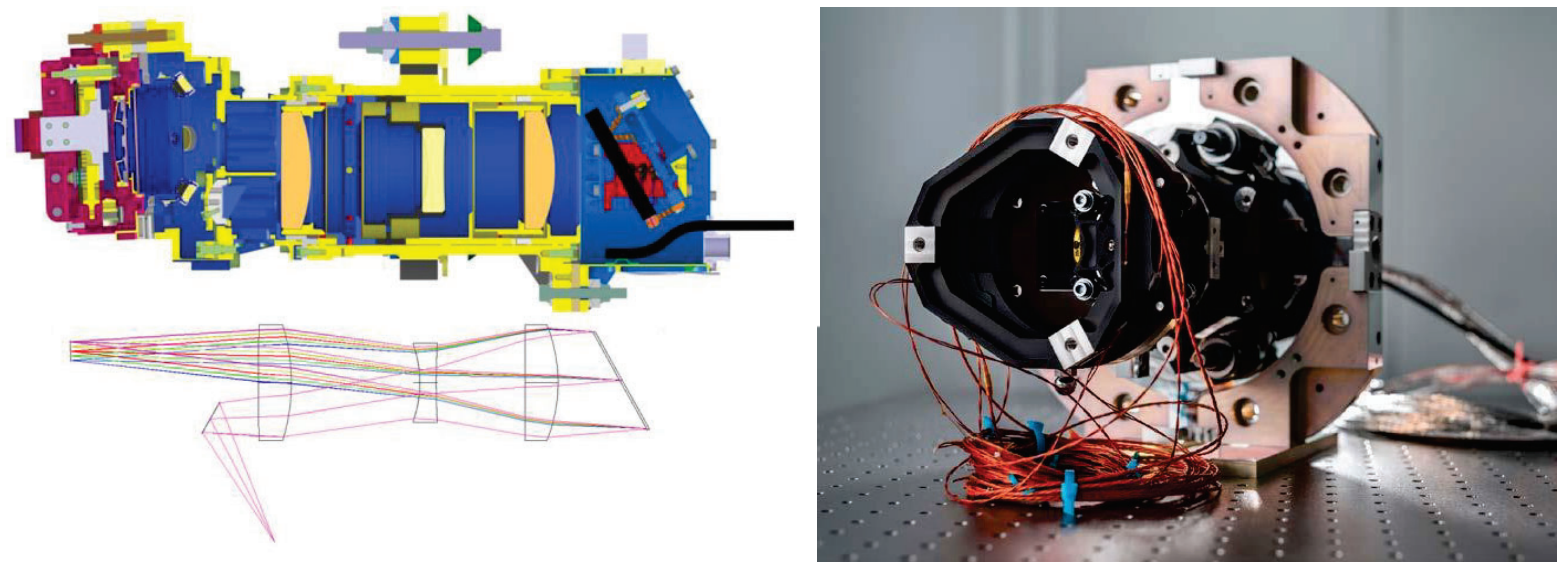

Figure 8. NIR Spectrograph Assembly (NSA) with NIR FPA. On the left side the mechanical crosss section (top view) and the optical layout (bottom view). The ray colors correspond to different wavelengths. On the right side a picture of the optical assembly without FPA (image credit: Florian Brill for Jena Optronik GmbH).

\subsection{Alignment steps}

The alignment and verification of the TSA can be divided in four main parts:

1. Integration and alignment of the COAs with the first characterization of the image space in air.

2. Second characterization of the image space with the FPAs in air.

3. Integration and alignment of FPAs to the vacuum position in air.

4. Alignment verification in vacuum.

In the first part of the alignment the three COAs are integrated and aligned in the TSA. In order to bring the telescope focus on the slit in laboratory conditions a defocus compensating the air-to-vacuum shift is computed by analysis and applied to the OGSE collimator (Figure 9). An accurate three dimensional characterization of the two optical image spaces is performed in laboratory conditions by means of the OGSE CCD camera with $9 \mu \mathrm{m}$ pixel pitch. The camera is scanned through the focus acquiring a set of test images with $10 \mu \mathrm{m}$ step. The image processing algorithm extracts the key parameters from each picture and fits them in the three dimensional space, thus allowing the definition of the best image plane location targeted by the FPA alignment. The relative position of TA to USA is aligned and frozen at first. The NSA ideal position is defined afterwards, taking also into account the measured as-built focal length of the UV-VIS channel. This approach allows building the instrument with equal end-to-end focal length for the two bands, a condition necessary to meet the challenging inter-band spatial co-registration requirements.
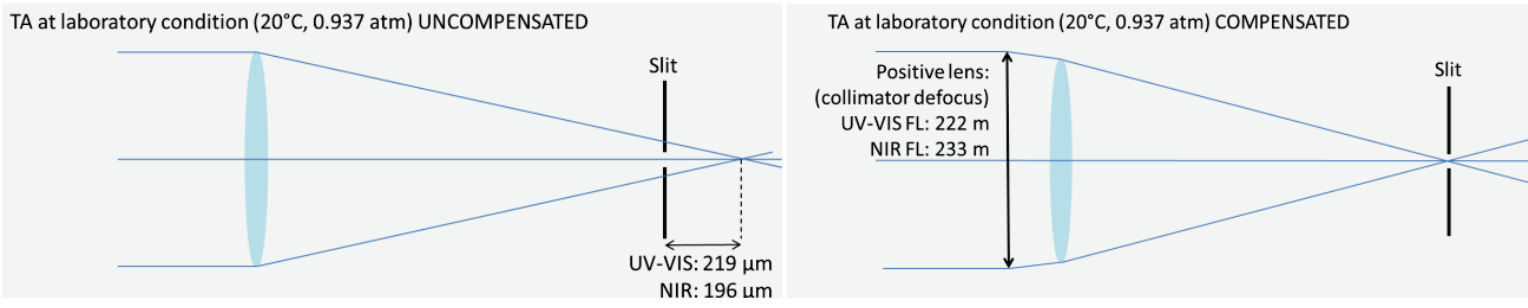

Figure 9. Schematic view of the TA working in air. The slit is aligned to the telescope focus relative to an object at infinity in vacuum. On the left: when operated in air without compensation the focus is shifted after the slit. On the right: a collimator defocus can bring the focus at the slit position in air. 
In the second part of the alignment the position of the two FPAs must be aligned to the best image plane location defined in the first part. The relatively large pixel size of the flight detectors $(27.5 \times 15 \mu \mathrm{m})$ does not allow an accurate check of the best image plane position relative to the detector surface with a single image acquisition. Therefore a specific GSE tool was developed in order to allow the scan of the FPA through the image space with the same steps previously performed with the OGSE CCD (Figure 11, left). The image processing algorithm is then computing the position of the FPA with respect to the best image plane in laboratory conditions. This position is later taken as reference in order to obtain the alignment of the FPA mounted on the shim in operating conditions after the application of the air-to-vacuum compensation.
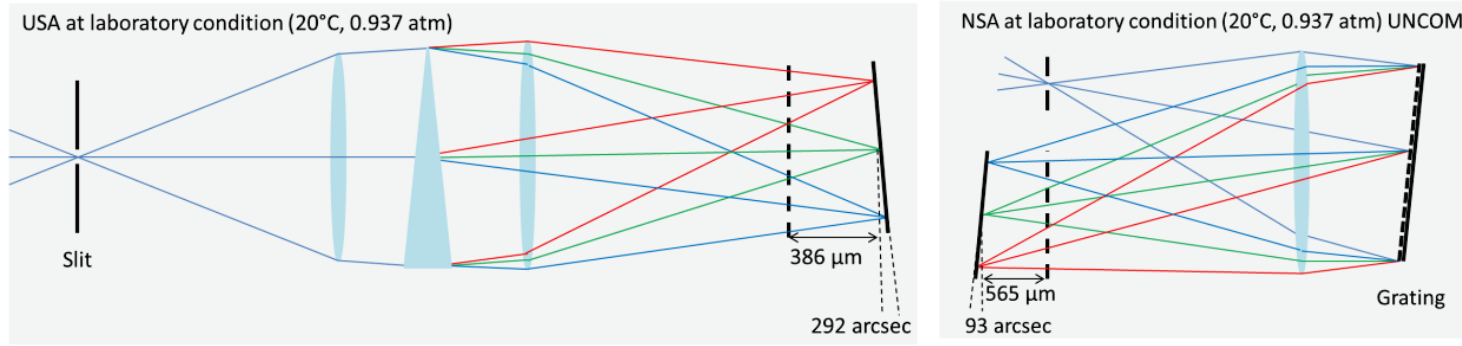

Figure 10. Schematic view of the UV-VIS (left) and NIR (right) spectrographs operated in air. When operated in air the focal planes are shifted away from the spectrograph and slightly tilted. The dashed lines indicate the nominal position of the focal plane in vacuum.

The best image plane position in operating conditions, due to the air-to-vacuum transition, is different from the position characterized in the first part of the alignment in laboratory conditions (Figure 10). Once the FPA position relative to the best image plane is characterized in laboratory conditions the air-to-vacuum shift computed by analysis is used to define and manufacture the alignment shims of the two FPAs.

In the third and last part of the alignment the two FPAs are mounted on the flight shim and the image produced by the OGSE, which is heavily blurred due to the air-to-vacuum shift, is used to align the lateral position and clocking of the two detectors. In order to meet the challenging spatial co-registration requirements for the accurate clocking of the FPAs a special alignment tool was developed and built (Figure 11, right).
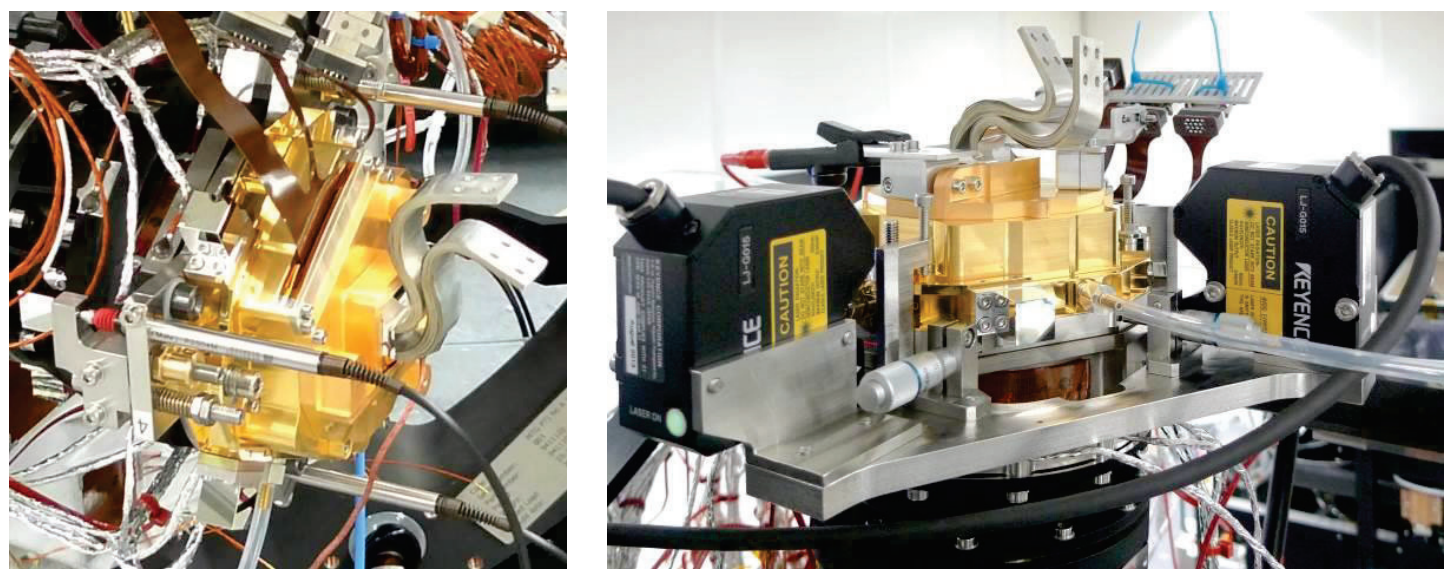

Figure 11. FPA alignment with specifically developed tools. On the left side the FPA is scanned through the image space in air during the second part of the alignment. On the right side the final lateral adjustment and clocking is performed with the FPA mounted on the flight shim.

As last step, after completing the alignment in laboratory, the TSA is moved to a thermal vacuum chamber for the final test in operating conditions (Figure 12). The OGSE used for the alignment is moved in front of the chamber and provides 
the input source through a window (Figure 14). The goal of the measurements in the chamber is to verify and characterize the TSA alignment status in operating conditions. If deviations requiring a correction are observed, the TSA can be moved again to the laboratory for an alignment iteration. In such case the measurements performed in vacuum provide the reference for the delta-alignment.
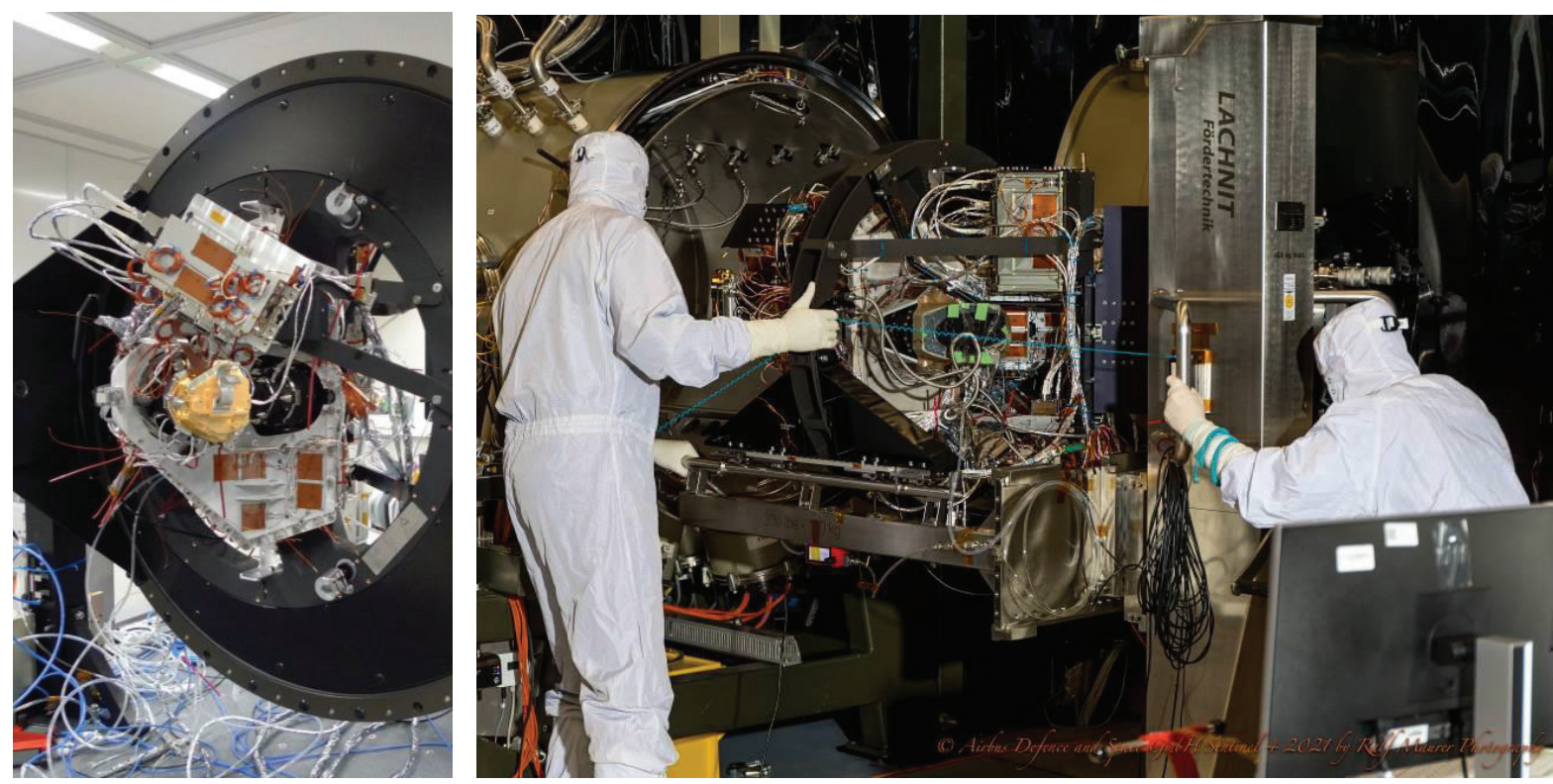

Figure 12. Left: TSA mounted in the alignment dolly at the end of the alignment in the ISO5 laboratory. Right: TSA in front of the TVAC chamber in preparation of the final test in operating conditions.

\section{OPTICAL GROUND SUPPORT EQUIPMENT}

The S4-UVN OGSE architecture is developed by MICOS and is based on a collimation module, two sensor modules and the control unit.
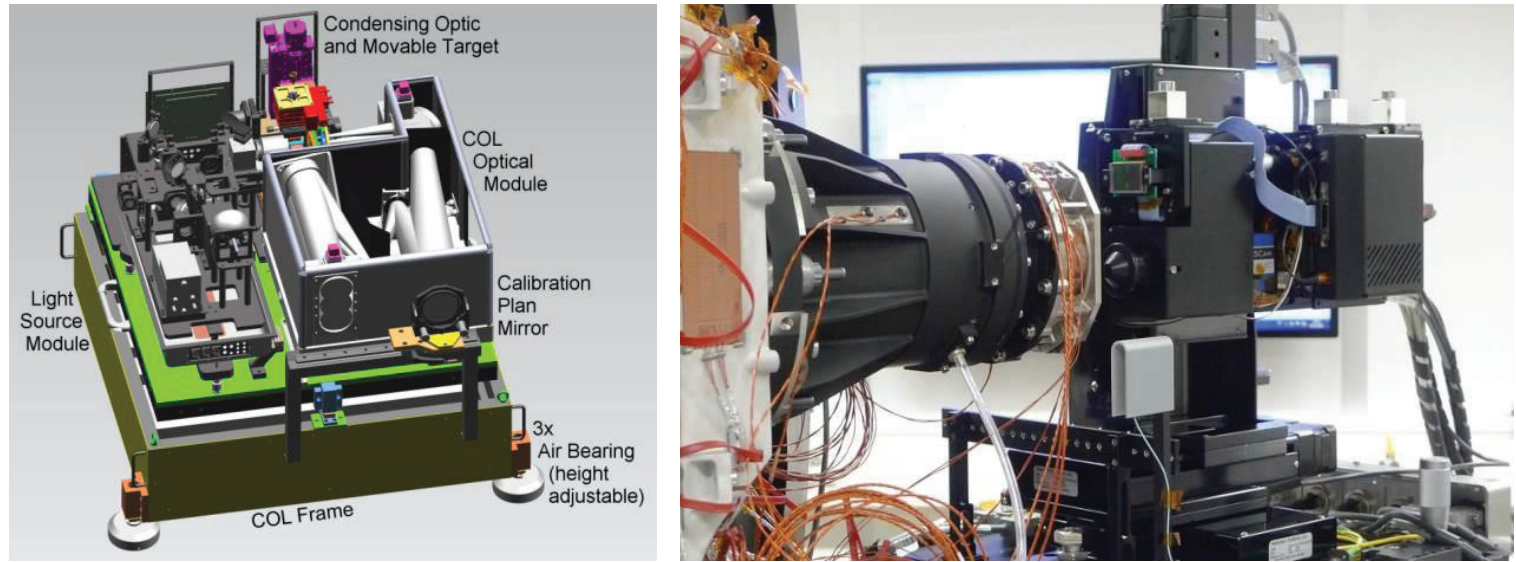

Figure 13. On the left side schematic view of the AIT OGSE COL module. On the right side picture of the sensor module with CCD detector and Shack-Hartmann sensor placed at the UV-VIS spectrometer image plane. 
The collimation module (COL) includes the focal plane moving object targets, some light sources with the condensing optics, a focusing tool (FOT) and mechanism for tip/tilt adjustments (Figure 13, left). The two sensor modules are optimised for UV-VIS and NIR spectral bands. Each module has a XYZ linear stages mechanism, a Shack-Hartmann sensor with collimating optics and an imaging camera (Figure 13, right), a large CCD camera sensor (without cover glass). The control unit includes the computer, the mechanism controllers, several fibre coupled light sources and the despeckle device.

All the equipment and sensors are used during the TSA alignment in laboratory, while only the COL module is used during the test in the TVAC chamber as the instrument flight detectors are already integrated (Figure 14).

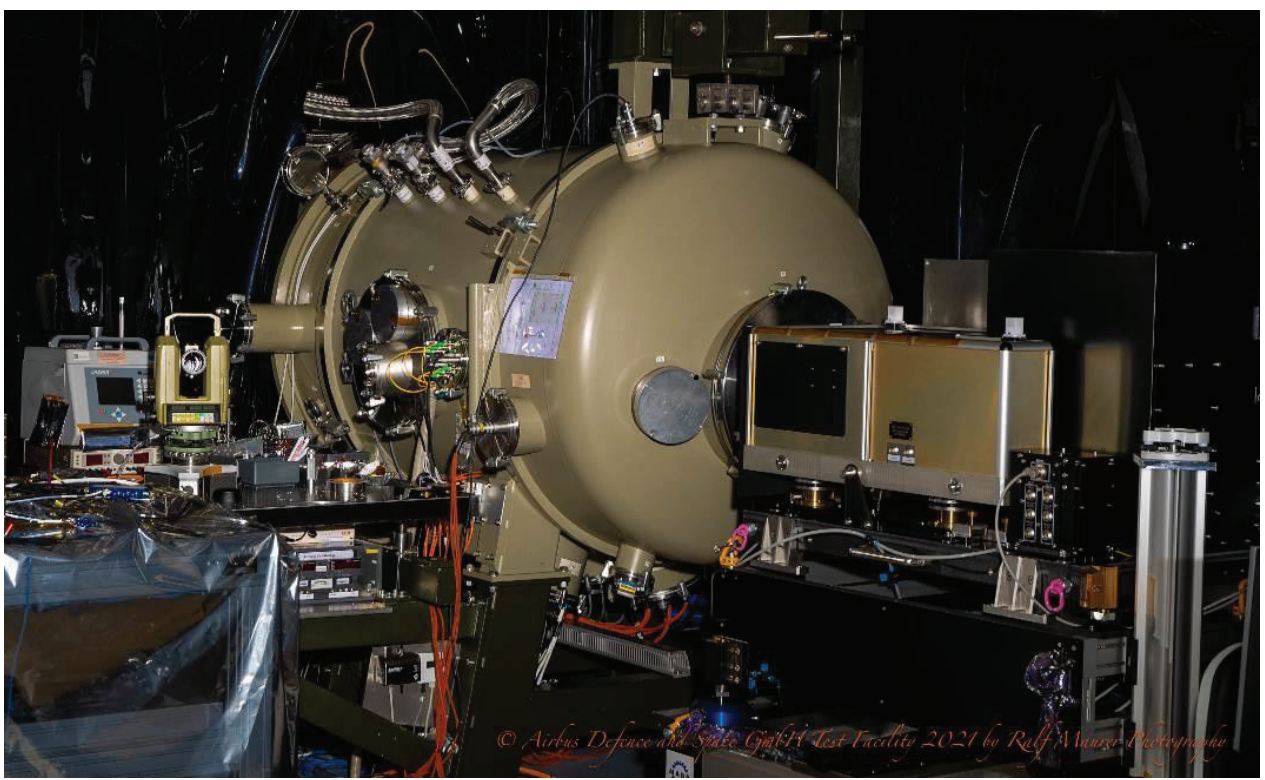

Figure 14. OGSE collimation module in front of the TVAC chamber. The fully aligned TSA is inside the chamber and is illuminated by the OGSE collimator through a fused silica window.

\subsection{COL module with focusing tool}

The optical design of the COL module consists of five mirrors (one folding mirror and four imaging mirrors). The design is telecentric at the object plane with a non-corrected intermediate image and with a real stop aperture in front of a mirror. The exit pupil is $1400 \mathrm{~mm}$ in front of the COL housing (the long distance is needed for illuminating the TSA entrance pupil during the thermal vacuum tests, as shown in Figure 15). The COL module is mounted on air bearing foots. The coarse adjustment (Rot-X, Rot-Y and Rot-Z) is performed with the air bearing foots and the theodolite measurements on the alignment cubes. The fine adjustment (Rot- $X$ and Rot-Z) is performed with the intermediate kinematic mount. The final adjustment of the targets in front of the S4 instrument is done with the target fine positioning during the first live view imaging with the detectors.

The COL module target unit has an additional mechanism with an individual light source and a wavefront sensor (ShackHartmann). In front of the COL module, a calibration plan mirror can be shifted in front of the COL beam. The focusing tool is used for setting the COL focus at infinity during the ambient measurements and before the thermal vacuum test for compensating any focus shift produced by the entrance window (used with a plane mirror placed in the TV chamber). 


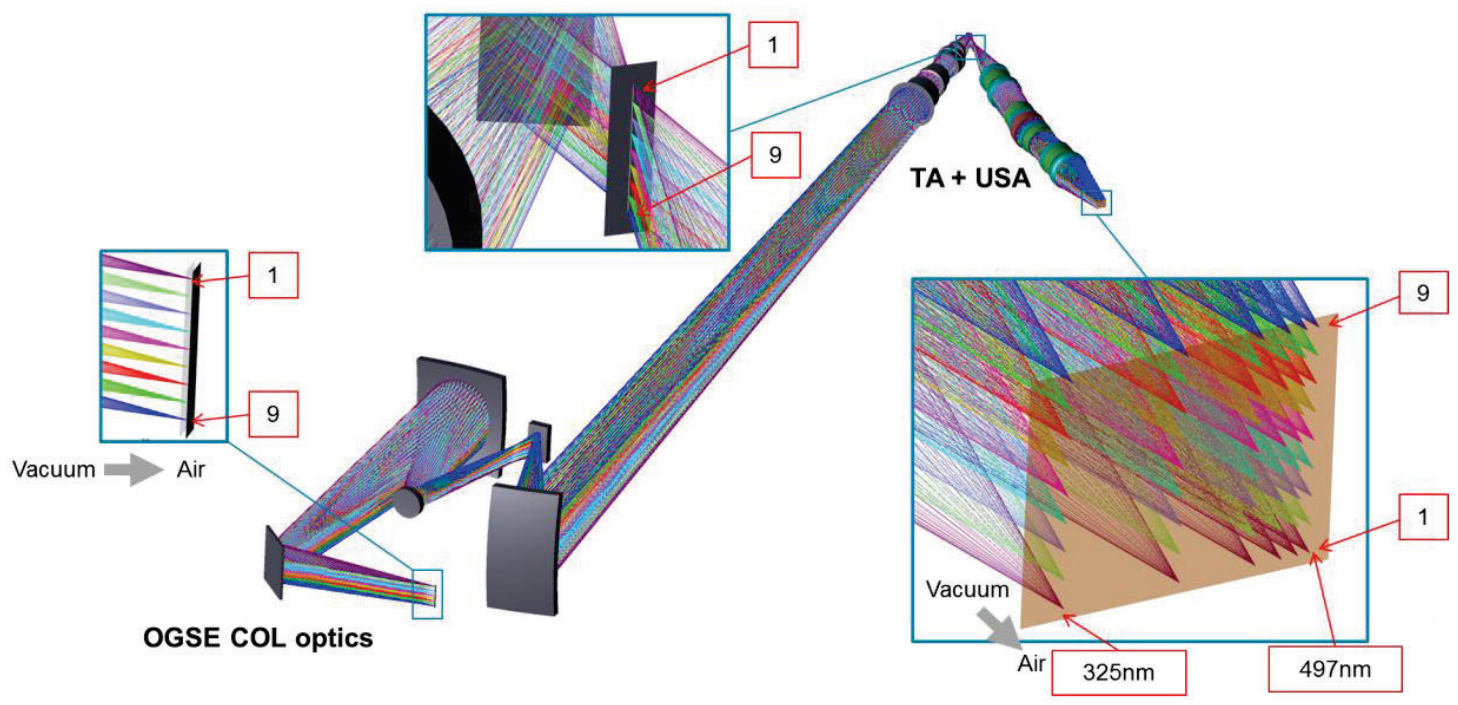

Figure 15. Optical layout of the AIT OGSE COL illuminating the TA and USA in test configuration. The layout shows how the COL focus and the image sensor positions are adjusted to compensate the air to vacuum effect.

\subsection{Light sources and object targets}

The light sources with glass fibre coupling are mounted in the control unit rack. Several laser sources are available in the UV-VIS range: HeCd UV laser (325 nm), Toptica laser $(405 \mathrm{~nm})$ and Ar-ion laser (several lines between 450 and 500 $\mathrm{nm}$ ). One laser source is available in the NIR range: Sacher laser (tuneable between 752 and $775 \mathrm{~nm}$ ) and for the full wavelength range an Eq99 continuum source (fibre coupled).

The flat field illumination sources are placed directly in the COL module: Argon emission lamp, Mercury emission lamp and the continuum source (Xenon arc lamp).

The object targets consist of a metal foil (laser cut) mounted between 2 holding plates. The targets are 9 pinholes in a row and several flat field targets: a slit, 5 small squares in a row and the focussing pattern (zebra lines). Each laser sources is usable with all the targets. The flat field sources are only usable with the flat field targets. The object targets are mounted on a XYZ mechanism for a target exchange and for the fine target positioning.

The use of monochromatic light sources with flat field illumination or continuum white light sources with object targets produces respectively a set of vertical or horizontal patterns on the spectrograph focal plane that are used to characterize the relevant geometrical optical performance parameters (Figure 16).
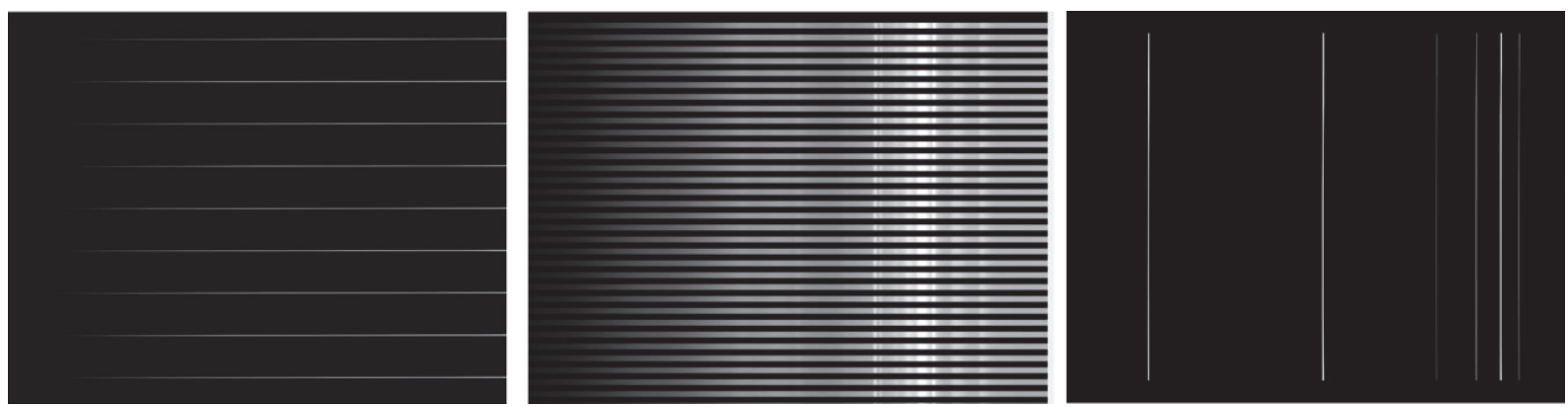

Figure 16. Three possible combinations of object targets and light sources used during the alignment at the USA focal plane. From left to right: Eq99 with pinholes, Eq99 with focusing pattern, flat field illumination with UV-VIS lasers. 


\section{IMAGE PROCESSING ALGORITHM}

A dedicated image processing algorithm is developed and used to calculate geometrical parameters (best focus position, centroid coordinates) and optical performance parameters (enslitted energy in spatial and spectral direction, distortion) from the images acquired during the alignment campaign. Objective of the algorithm is to provide the input parameters necessary to define the alignment steps and to finally judge the alignment status.

The main algorithm capabilities are:

1. Automatic detection of the region of interest within imported optical images.

2. Determination of the best image plane (BIP) location from a set of optical images taken during a through focus scan (detector movement along the spectrographs optical axis).

3. Three dimensional characterizations of the relevant optical performance parameters in the image space of the spectrometers.

4. Computation of alignment parameters (6 degrees of freedom) with respect to a physical detector reference position.

Through focus scans are performed in different phases of the alignment either with the AIT-OGSE CCD and/or the FPA detector. During the test in the TVAC chamber the FPA detector position is fixed but the scan is performed moving the COL object target along the optical axis.

\subsection{Determination of the best image plane location}

A series of optical parameters are extracted from the images recorded at each detector position in the through-focus scan.

Spectral and spatial enslitted energy parameters (described in the next paragraph) in combination with $\mathrm{X}$ and $\mathrm{Y}$ centroid coordinates are computed for each scanning position thus creating a detailed three-dimensional characterization of the image space. This information is used to define the location of best image plane (BIP), i.e. the planar surface in the image space delivering the best overall performance. The algorithm computes also the performance on such surface by fitting the data recorded at each scanning position (Figure 17).

It is clear that the BIP position is not defined unambiguously but will be dependent on the weight given to different performance parameters. As an example the slit alignment accuracy inside the TA and the longitudinal chromatic aberrations of the dioptric design generate a shift between the best image plane for spectral and spatial performance. In this case the goal of the alignment is to bring the flight detector in the position that delivers the best overall performance taking into account all the instrument performance budgets. The accurate three dimensional characterization of the image space provides therefore all the inputs necessary to identify the optimum alignment target for the FPAs.

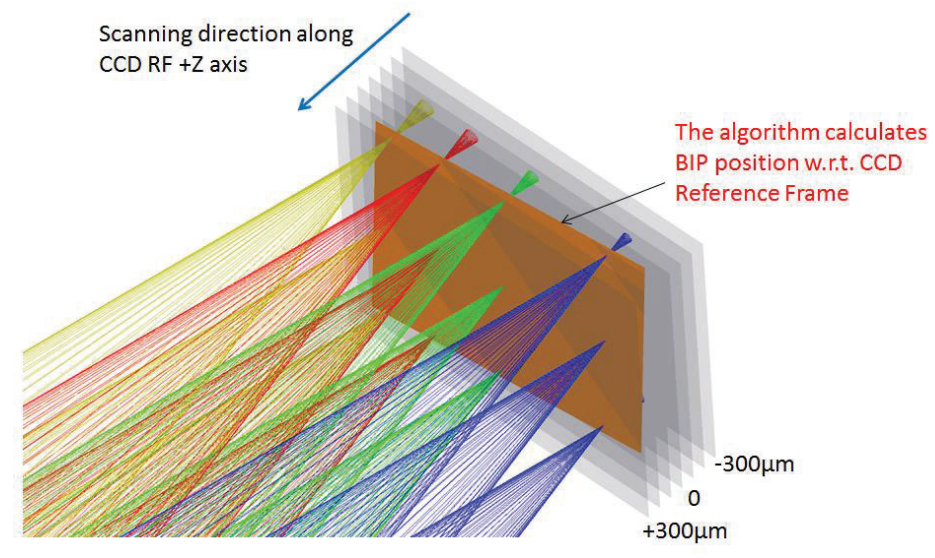

Figure 17. Schematic view of the through-focus scan measurement. The detector is scanned through the image with equally spaced steps and several test images are recorded for each position. The algorithm computes the best image plane (BIP) location defined in the scanning reference system. 

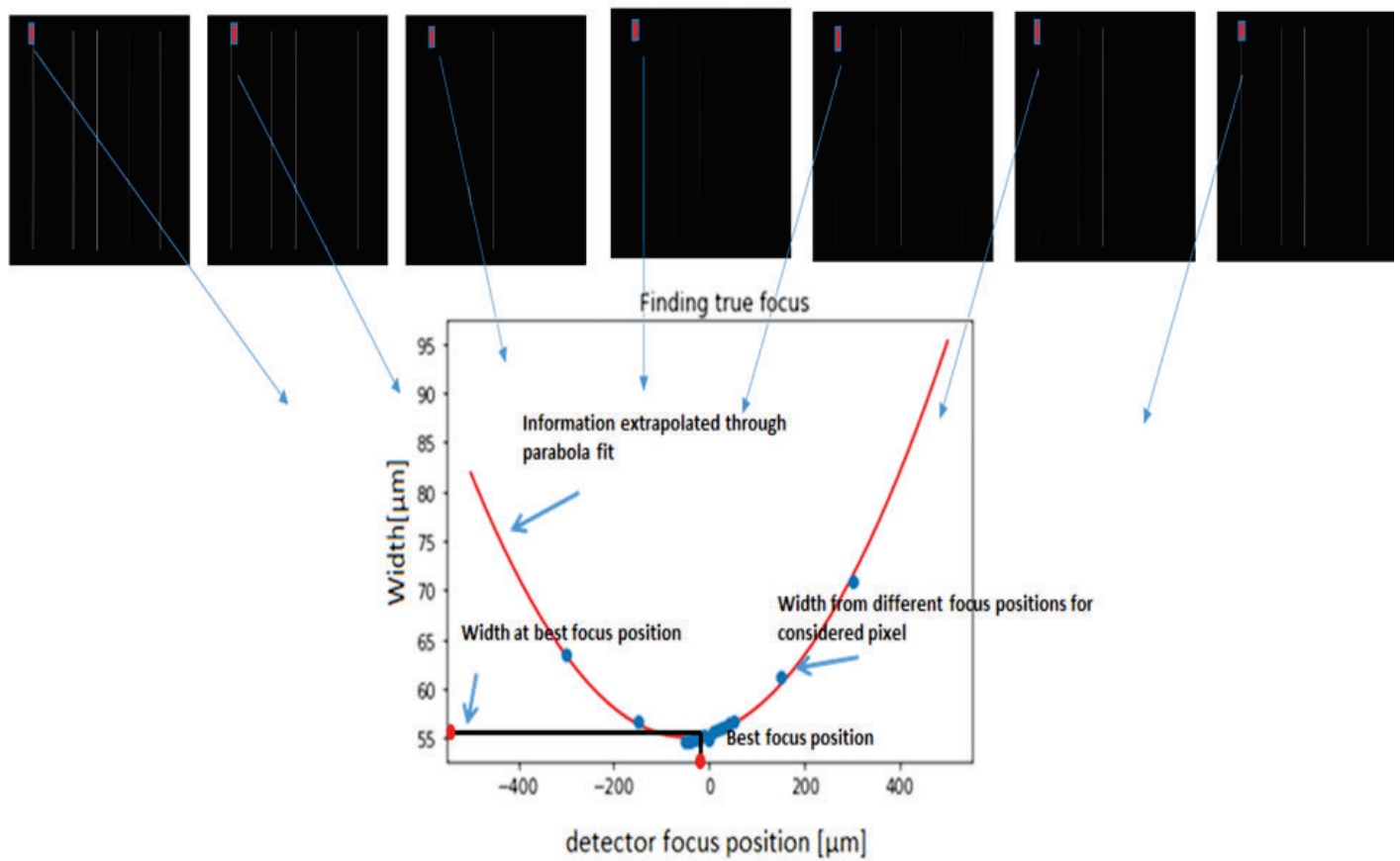

Figure 18. Plot showing the algorithm fit in the calculation of the best focus axial coordinate. The fit is performed extracting the enslitted energy value for a point of the image at each scanning position. In the example above the spectral enslitted energy is extracted from a monochromatic wavelength at the top-left corner of the image and the best focus coordinate is computed for this point.

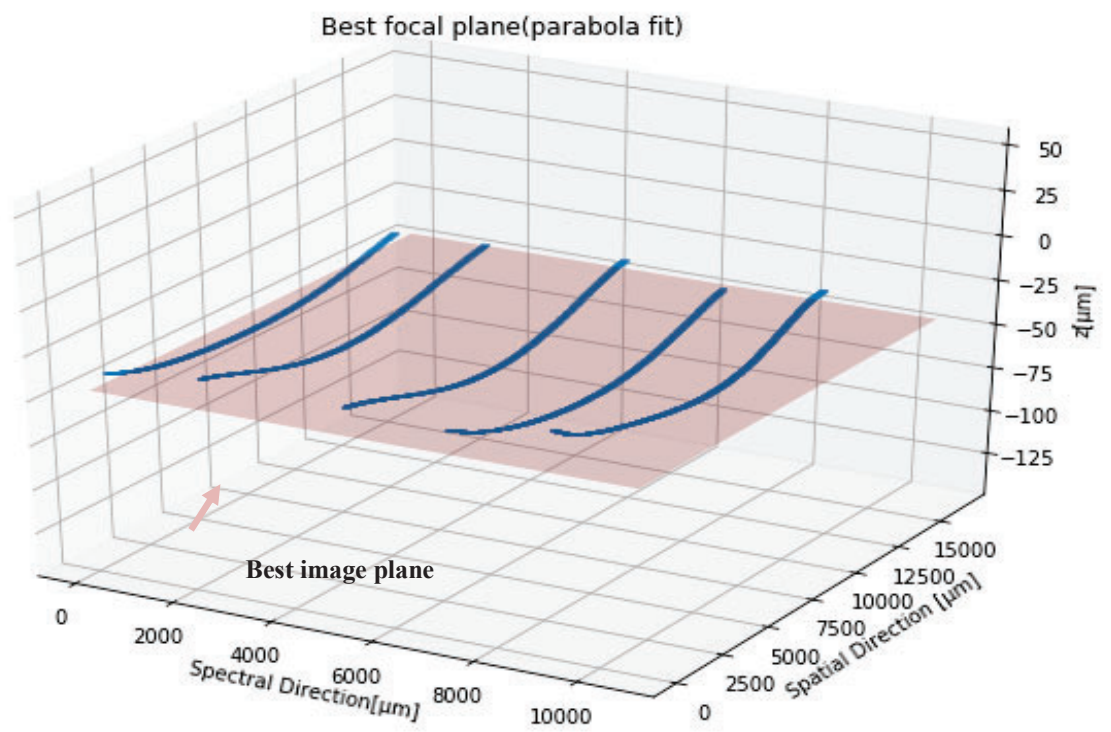

Figure 19. The best focus axial coordinate as a function of the spectral and spatial coordinate on the detector reference system is used to calculate with a least squares fit the best image plane location. 


\subsection{Spectral and spatial enslitted energy parameters}

One of the key optical performance parameters defining the image quality is the enslitted energy. This parameter can be defined along the spectral or spatial direction and is essentially defining the sharpness of the image. It is correlated to the optical PSF delivered by the optical subsystems in combination with the alignment of slits and detectors. Spatial enslitted energy is linked to the integrated energy performance requirements of the instrument in North-South while spectral enslitted energy to instrument spectral performance requirements. The instrument integrated energy performance in EastWest is entirely defined by the slits alignment inside the TA is not affected by the TSA alignment.

Along each line within the region of interest, the algorithm computes the width for a pixel column respectively along the spatial or spectral direction for slit or focusing pattern images. The data extracted from the images are fitted with the convolution of a top hat function (a box) modeling the slit or the focusing pattern and a Gaussian function modeling the optical point spread function (PSF). From the fitted curve the algorithm is then computing the full width at quarter maximum (FWQM) minus full width at three quarters maximum (FWTM) which is found to best correlate to the spatial enslitted energy parameter. The FWQM minus the geometrical width of the slit image is computed for the spectral enslitted energy parameter (Figure 20).

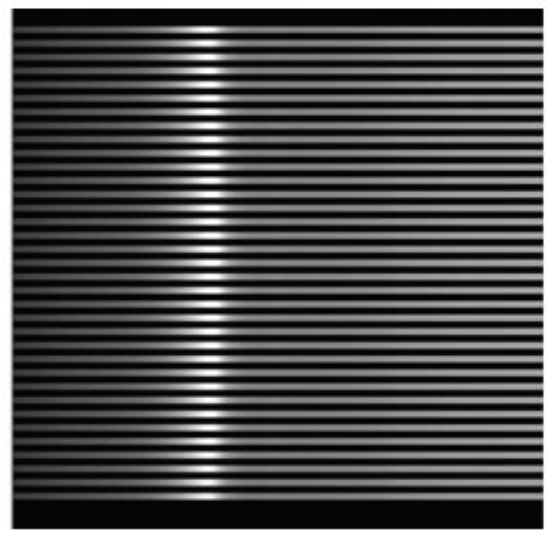

- Information from real image

Information extrapolated after fermi fit
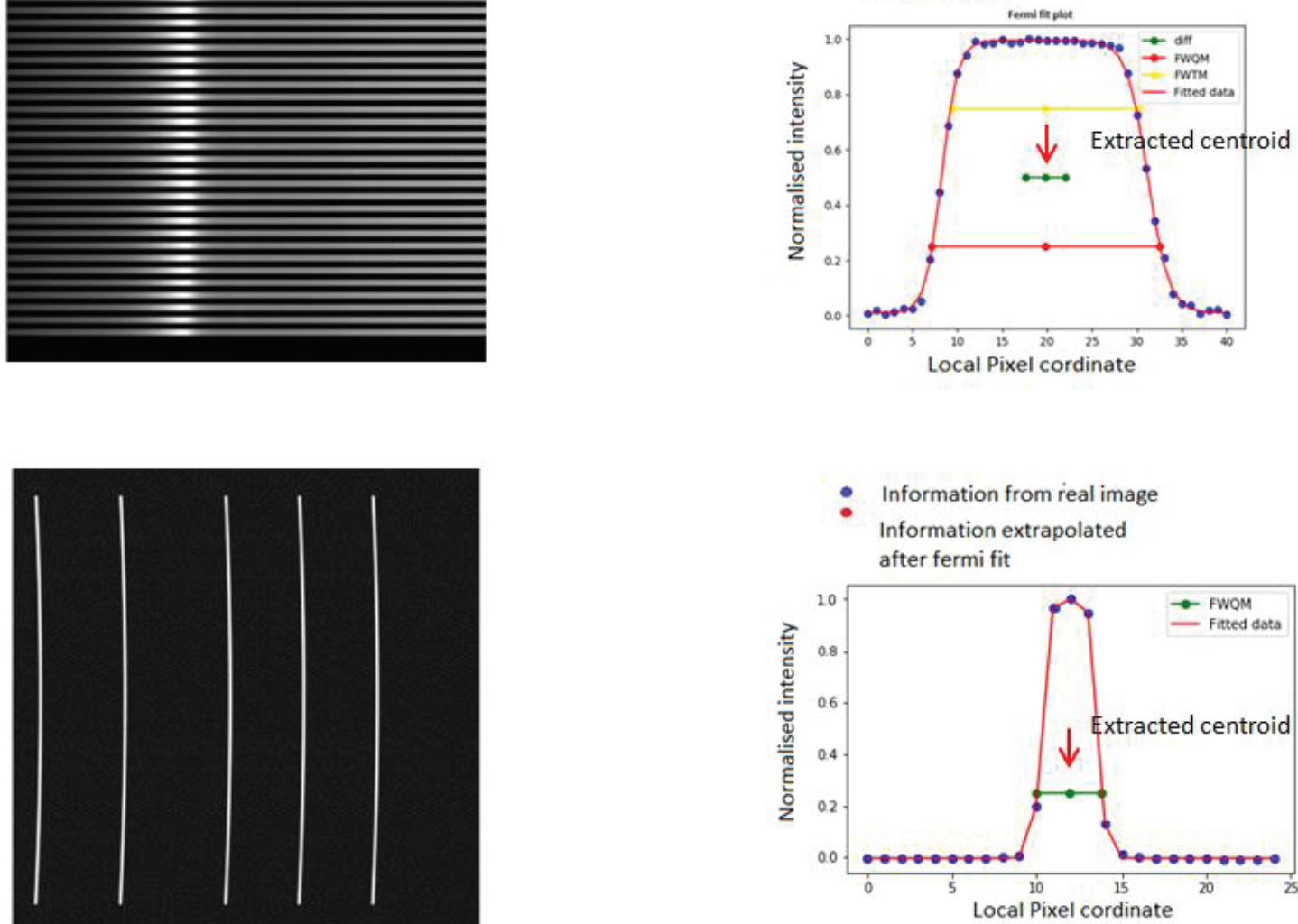

Figure 20. Calculation of spatial and spectral enslitted energy parameters. On the top the spatial enslitted energy is extracted from the focusing pattern image with white light source. At the bottom the spectral enslitted energy is computed from the monochromatic slit images.

\subsection{Centroid coordinates and clocking}

For each point of the focusing pattern lines and the monochromatic slit images the algorithm is computing the centroid coordinate. Spectral centroid coordinates are used to define the image envelope for the nominal wavelength range and 
determine the lateral alignment of the FPA. Spatial centroid coordinates are used to calculate distortion and coregistration parameters as explained in the next section.

From the focusing pattern images the algorithm is also able to calculate the image clocking angle as shown in Figure 21. The clocking angle for each of the focusing pattern lines is computed from the spatial centroid coordinates. The average value is computed and used to determine the target FPA clocking angle in the last part of the alignment.

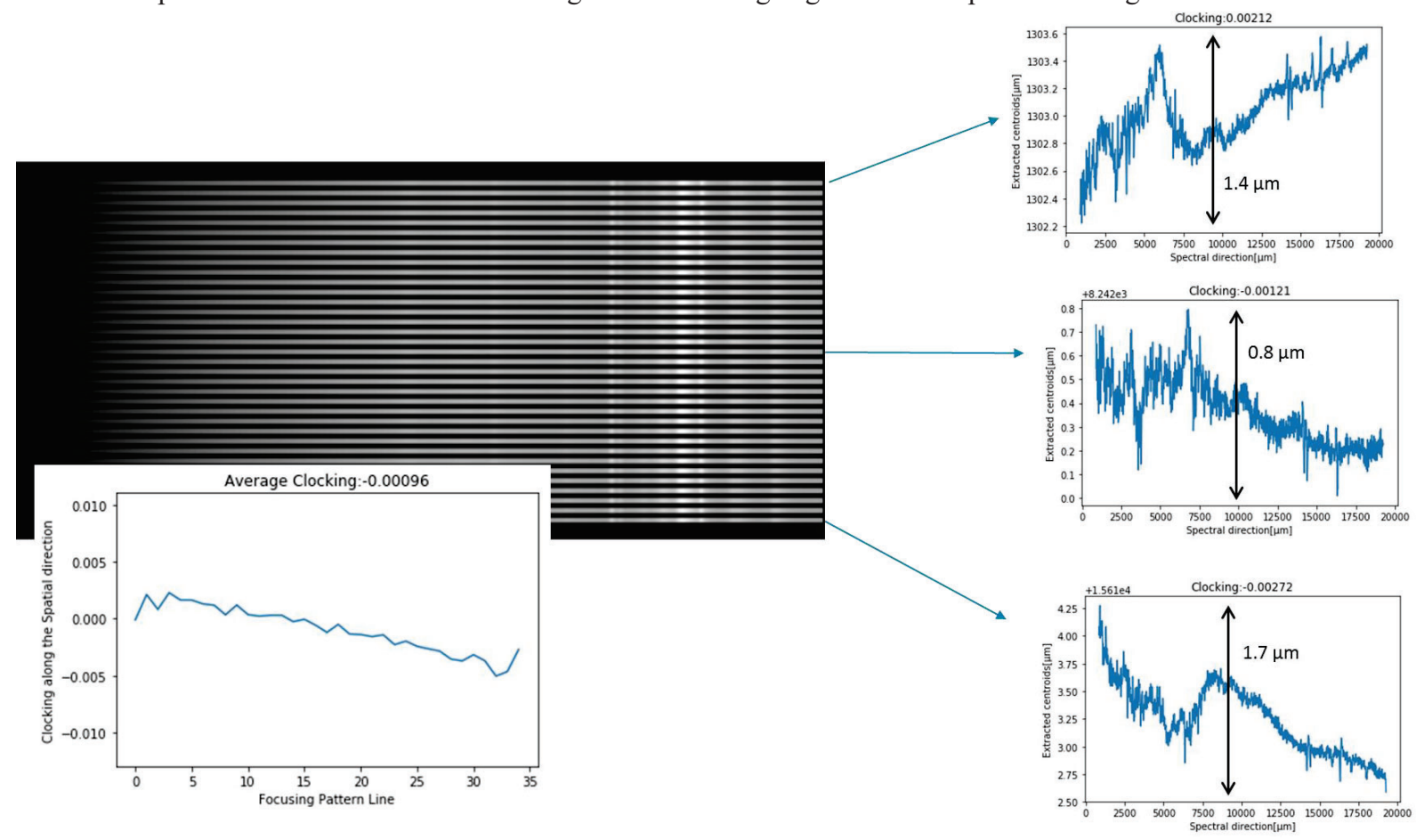

Figure 21. Clocking angle computed from the focusing pattern image with white light source. On the right side three plots with the spatial centroid coordinates for the top, middle and bottom focusing pattern line (with different scale). On the left side a plot of the clocking angle for each line from top to bottom. In this specific case the detector is aligned to the image with better than 4 arcseconds accuracy, which indeed is the final alignment of the UV-VIS FPA in air.

\section{OPTICAL PERFORMANCE PARAMETERS OVERVIEW}

The TSA alignment verification is based on the evaluation of four optical performance parameters:

1. Optical PFS in spatial (N/S) direction (linearly related to the spatial enslitted energy parameter described in the previous section).

2. Optical PFS in spectral direction (linearly related to the spectral enslitted energy parameter described in the previous section).

3. Intra-band distortion.

4. Inter-band co-registration.

As explained in the previous section, centroid coordinates and parameters of spatial and spectral enslitted energy are extracted from the images processed by the algorithm. From this information the optical performance parameters are computed in order to evaluate and assess the system alignment status and estimate its performance. 


\subsection{Optical PSF}

In order to quantify the optical PSF the following assumptions are made:

- $\quad$ Optical PSF size in spatial $(\mathrm{N} / \mathrm{S})$ direction $\approx$ spatial RMS Spot Size

- $\quad$ Optical PSF size in spectral direction $\approx$ spectral RMS Spot Size

To properly assess the enslitted energy parameters extracted from the test images, a conversion from these parameters to the spatial and spectral PSF (spatial and spectral RMS spot sizes) was done in the optical model.

Zemax Opticstudio software was used to simulate some realistic test images produced from a series of perturbed systems: simulations were done for different detector focus positions. For each position:

- $\quad$ The native spatial and spectral RMS spot sizes of the perturbed system were computed.

- $\quad$ Focusing pattern and slit images were simulated from the perturbed systems.

- Spatial and spectral enslitted energy parameters were computed by the algorithm from the simulated images.

Comparing the RMS spot sizes with the enslitted energy parameters a linear relation between them was established (see Figure 22).
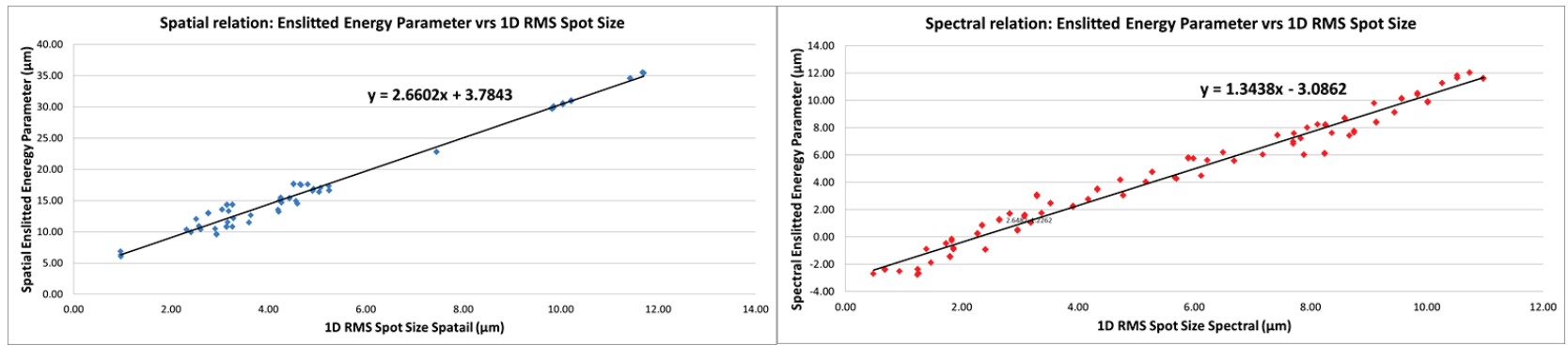

Figure 22. Correlation between Enslitted Energy parameters and RMS Spot Size (left: spatial correlation; right: spectral correlation)

In order to assess if the required alignment is achieved, a success criterion for the optical PSF is defined. The End-ofLife allocations of the spatial and spectral RMS spot sizes are rescaled down to the status at the End-of-Alignment. This operation is done according to the distribution of the different contributors of the end-to-end tolerance analysis. The resulting success criteria for the spatial and spectral RMS spot sizes are converted to spatial and spectral enslitted energy parameters using the linear relation shown in Figure 22.

Figure 23 shows the measured spatial enslitted energy parameters over the full field of view (from $-2^{\circ}$ to $+2^{\circ}$ ) with the detector in its final position (left: UVVIS channel, right: NIR channel). Each vertical line corresponds to a different line of the focusing pattern image from top to bottom. The length of the lines gives the variation of the spatial enslitted energy value over each focusing pattern line.

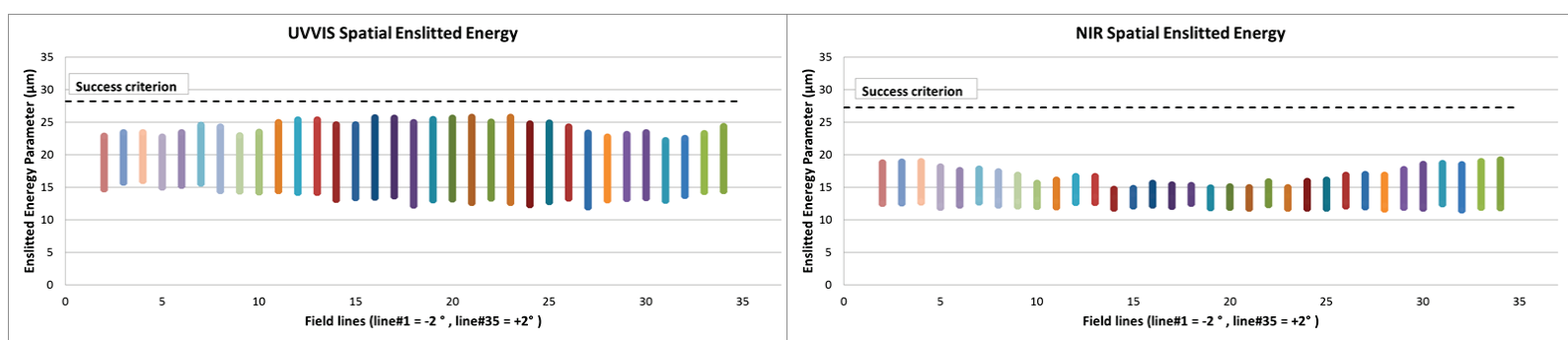

Figure 23. Spatial Enslitted Energy parameter (left: UVVIS channel; right: NIR channel) 
Figure 24 shows the spectral enslitted energy parameters with the detector in its final position (left: UVVIS channel, right: NIR channel). Each vertical line corresponds to a different wavelength inside the corresponding band. The length of the lines gives the variation of the spectral enslitted energy value over each wavelength.
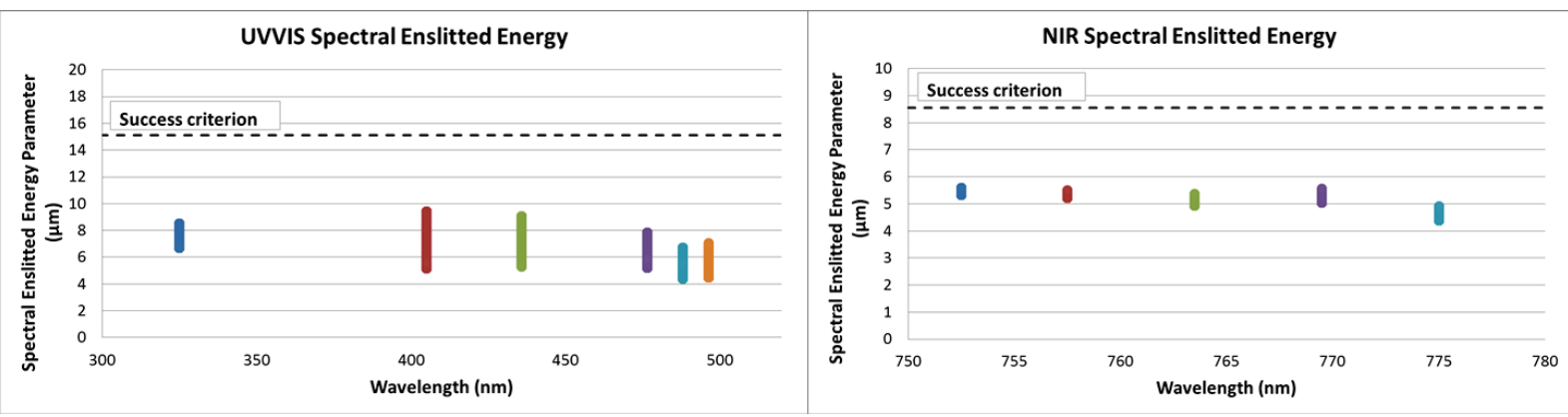

Figure 24. Spectral Enslitted Energy parameters (left: UVVIS channel; right: NIR channel)

\subsection{Co-registration and distortion}

From the N/S centroid coordinates the two following performance parameters are calculated:

- Intra-band distortion (for UVVIS and NIR):

Maximum peak-to-valley centroid coordinate variation in one field along the wavelength (for each channel independently)

- Inter-band co-registration:

Maximum peak-to-valley centroid coordinate variation between UVVIS and NIR channels in one field along the wavelength.

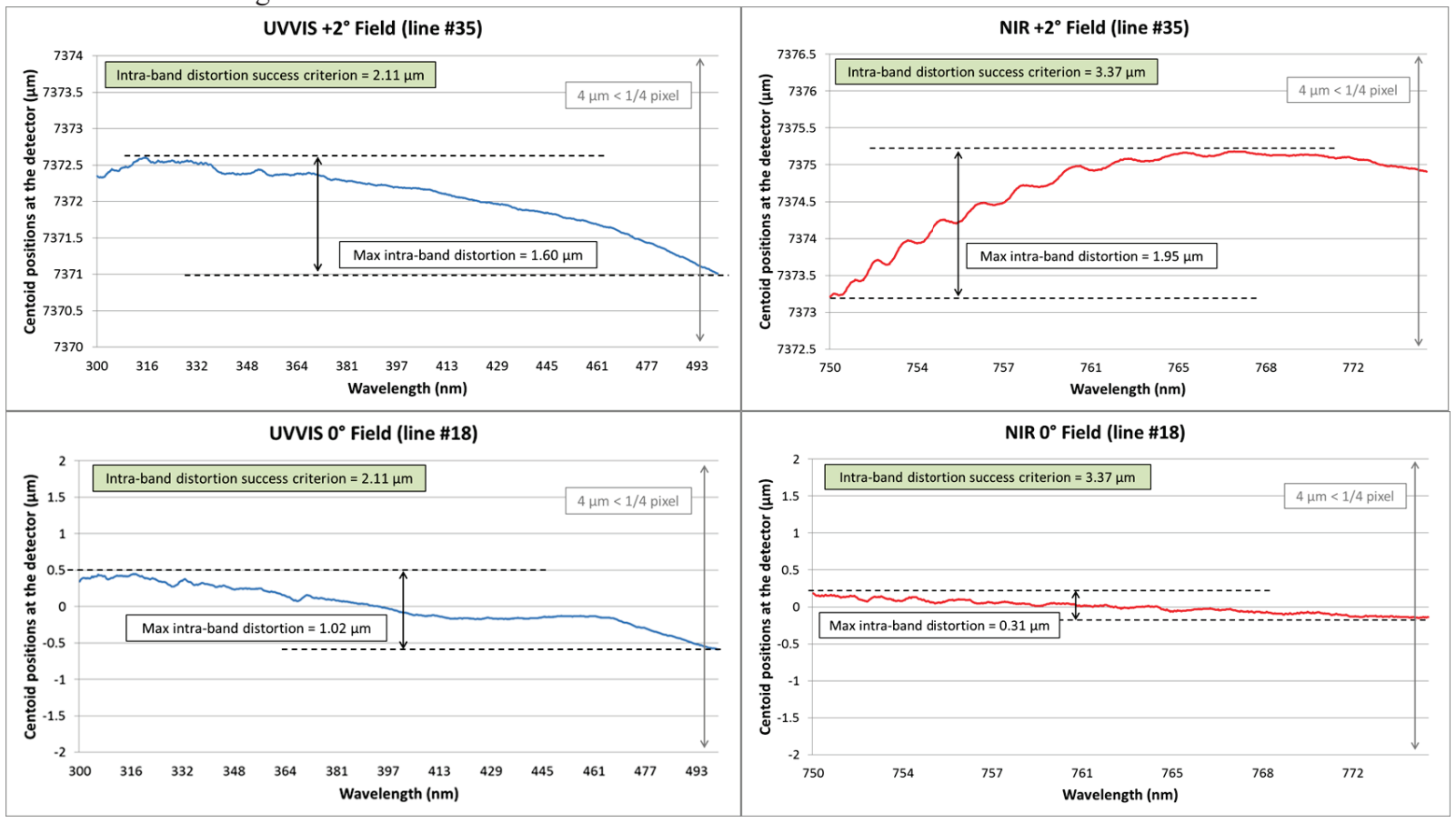

Figure 25. Field $0^{\circ}$ (top) and $+2^{\circ}$ (bottom) Intra-band distortion (left: UVVIS channel; right: NIR channel) 
In order to evaluate the quality of the alignment a success criterion for each parameter is defined. Again here the End-ofLife allocation is rescaled down to the End-of-Alignment status following the distribution of the different contributors from the end-to-end tolerance analysis.

Figure 25 shows the intra-band distortion at the field center $\left(0^{\circ}\right)$ and at the field edge $\left(+2^{\circ}\right)$ for each band (left: UVVIS, right: NIR). The measured values were all within the allocation also with considerable margin.

Figure 26 shows field $0^{\circ}$ and $+2^{\circ}$ inter-band co-registration (blue corresponds to the UVVIS channel, and red corresponds to the NIR channel).

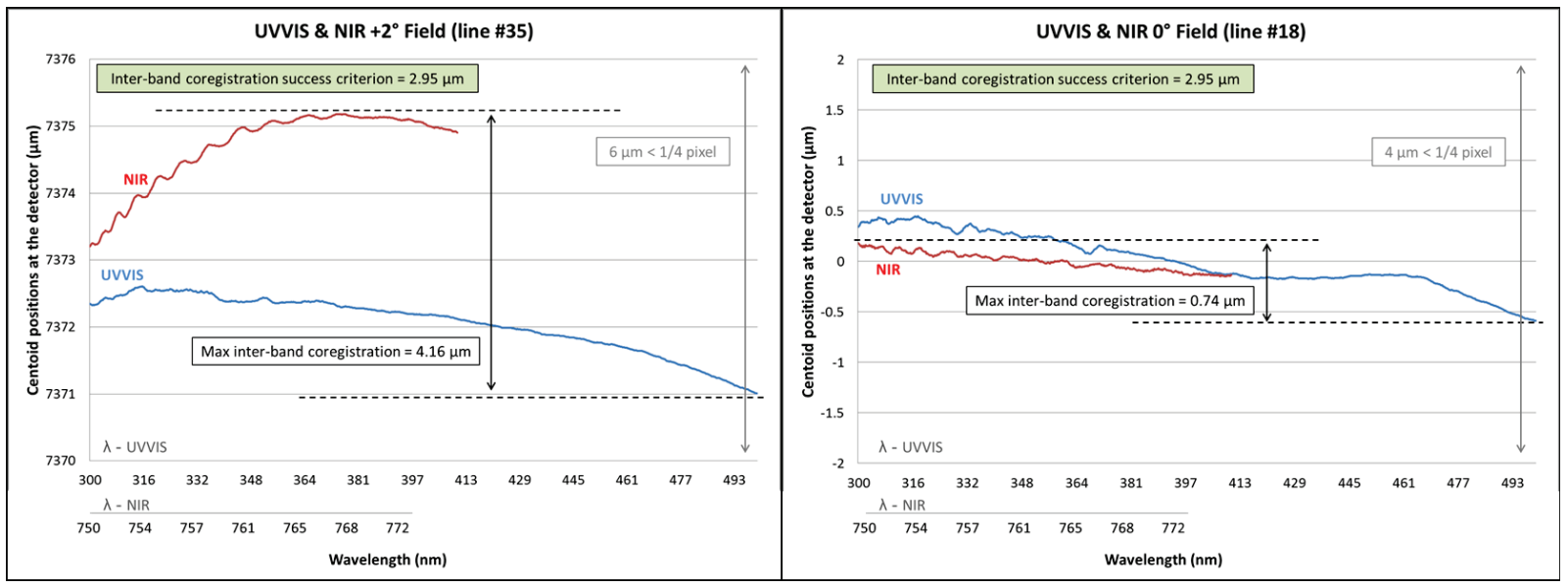

Figure 26. Field $0^{\circ}$ (right) and $+2^{\circ}$ (left) Inter-band co-registration (blue line: UVVIS channel; red line: NIR channel)

The spatial inter-band co-registration at instrument level is required to be less than $1 / 5$ th of a spatial element and the worst-case value measured at the edge of the field $(4.16 \mu \mathrm{m})$ compared to the spatial pixel pitch of $27.5 \mu \mathrm{m}$ is still less than $1 / 6$ th of a pixel (Figure 26, left side). Unfortunately the worst-case measured value is exceeding by approximately $1.2 \mu \mathrm{m}$ the success criterion that takes into account all the contributors and the margins for the future loads that the instrument will see until End-of-Life. The reason for this deviation is found in the different end-to-end as-built focal length ratio of the two channels that in the air-to-vacuum transition deviated marginally from the predicted behavior.

A correction of this deviation is technically possible but it would require a delta alignment with re-shimming of the NSA and NIR FPA. Due to the impact on the schedule and the risk of deteriorating other performance parameters, the project has decided to not perform the delta alignment. 


\section{OVERVIEW OF THE CURRENT STATUS AND CONCLUSIONS}

At the time of writing, the TSA alignment is successfully completed in one step without the need of alignment iterations. All the optical performance parameters are measured in operating conditions and are compliant to the alignment target values, with the exception of the marginal deviation measured for the inter-band co-registration discussed in the previous section.

The TSA PFM has been moved to the next facility for the integration inside the Optical Instrument Module which is already started and will be completed in Q1 2021. The final instrument performance verification is planned for the second half of the year during the calibration and characterization campaign at the RAL facilities, in the UK.

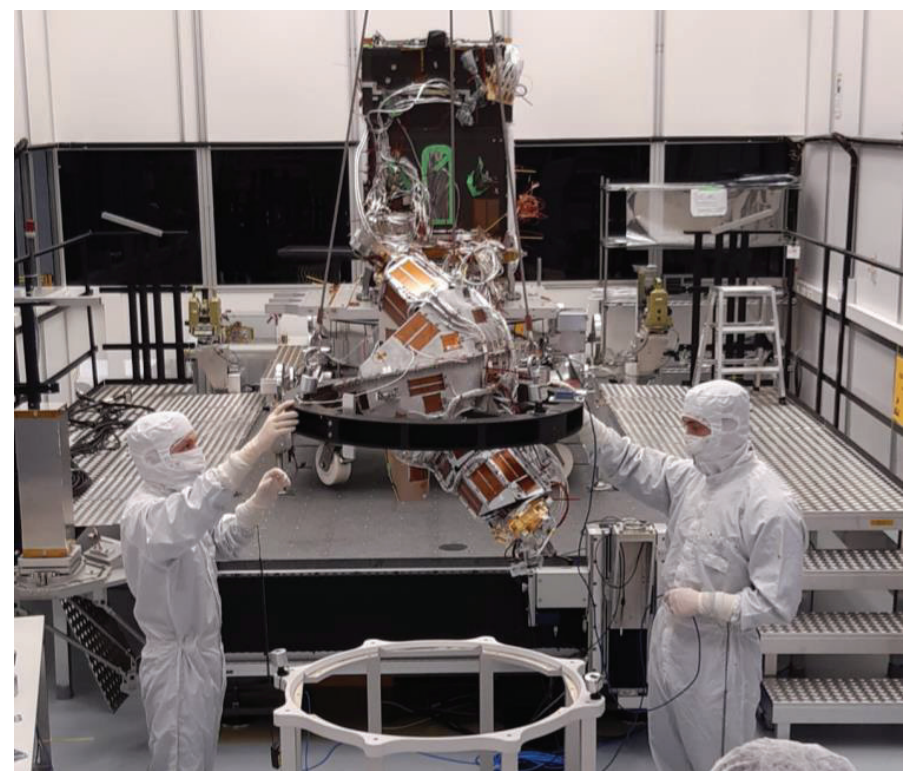

Figure 27. The fully aligned TSA after transportation to the cleanroom where it will be integrated inside the Optical Instrument Module (visible in the background)

\section{ACKNOWLEDGEMENTS}

The authors would like to express their thanks to their respective colleagues at the European Space Agency and Airbus Defence and Space, as well as to all the partner companies within the Sentinel-4 industrial consortium for their valuable contributions to the continuing success of this very challenging program. This article has been produced with the financial assistance of the European Union. 